\section{No prolonged effect of Ankaferd Blood Stopper on chronic radiation proctitis}

Ankaferd Blood Stopper (ABS) is a Turkish folk-medicine herbal extract which forms a hemostatic web on bleeding areas by inducing erythrocyte aggregation [1]. We previously reported the first case of successful ABS usage in severe radiation colitis [2]. In this observational study, a total of eight patients with bleeding due to chronic radiation proctitis (CRP) were treated with endoscopic ABS application as a primary therapy. The lesions were severe in all cases according to Wachter's classification [3]. ABS was instilled onto the bleeding areas by sclerotherapy needle or heater probe catheter, once a week, at a dose of 20-30 ml per session. ABS-induced hemostasis lasted for 1-8 days per session, and was achieved in seven of eight cases ( $\bullet$ Fig. 1).

In the eighth case bleeding was only lessened. However, recurrence of bleeding was the rule and the ABS had no effect on telangiectasia at the last follow-up. So, its blood-stopping activity for bleeding telangiectasia is only temporary. However, it was found to be effective in healing radiation-induced ulcers $(n=4)(\bullet$ Fig. 2 ). Afterwards, six patients underwent argon plasma coagulation (APC; complete hemostasis in 5 , lessened bleeding in 1), 1 patient underwent successful heaterprobe coagulation, and 1 (patient no. 5) required no therapy.

The optimal treatment of bleeding due to CRP is still debated. Currently, APC and local application of formalin are being used as the main successful measures to treat CRP; APC appears safer than formalin [4]. ABS has a transient hemostatic effect lasting 1-8 days in bleeding due to CRP. It may well lead to apparent healing of ulcers, but it is not useful for healing of telangiectasia or as a definitive therapy for bleeding. So, its use is not recommended as a routine treatment for CRP.

Competing interests: None
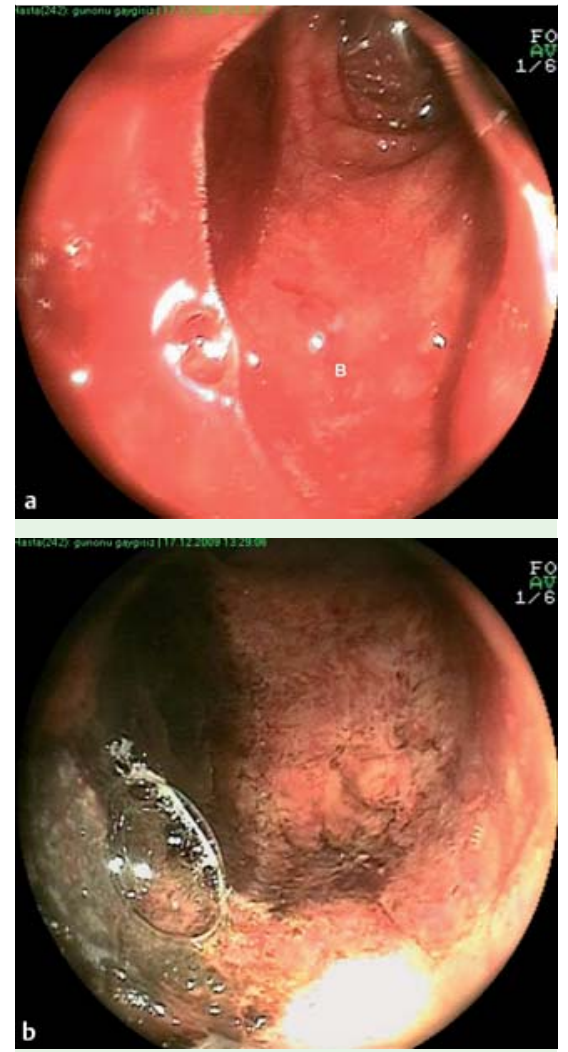

Fig. 1 a Diffuse oozing bleeding at the distal rectum from friable telangiectatic mucosa. b Grayish-yellow coagulum covered the diseased area within seconds after topical ABS application and bleeding stopped.

\section{Endoscopy_UCTN_Code_TTT_1AQ_2AZ}

E. Ozaslan ${ }^{1}$, T. Purnak ${ }^{1}$, G. Özyigit ${ }^{2}$, F. Akyol ${ }^{2}$, A. Yildiz ${ }^{1}$, I. C. Haznedaroglu ${ }^{3}$ Numune Education and Research Hospital, Department of Gastroenterology, Ankara, Turkey

2 Department of Radiaton Oncology, Faculty of Medicine, Hacettepe University, Ankara, Turkey

Department of Hematology, Faculty of Medicine, Hacettepe University, Ankara, Turkey
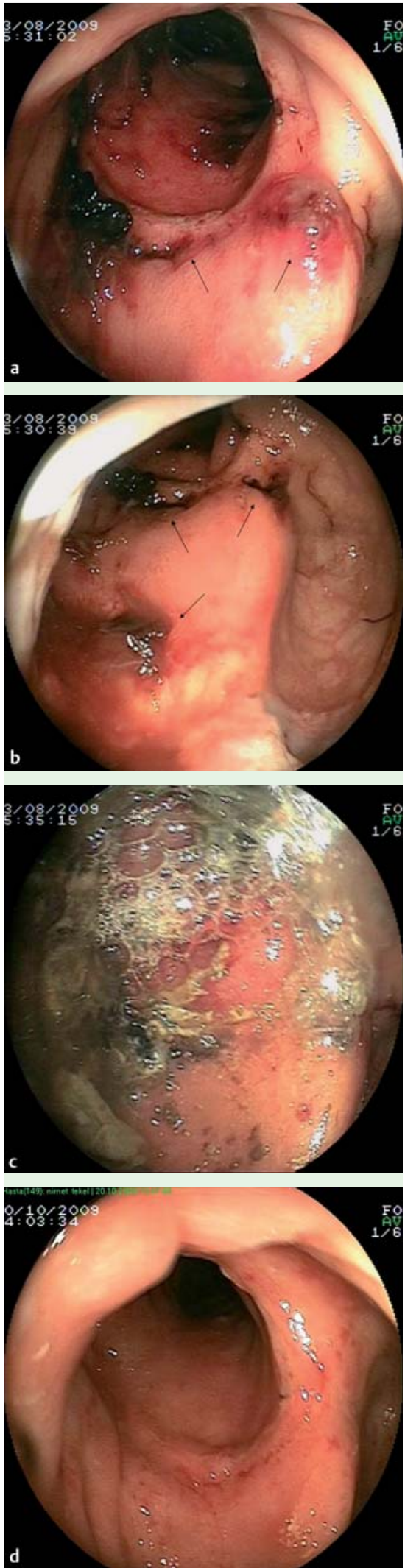

Fig. 2 a, b Ovoid and linear ulcerations with some clots at and near the anastomosis (center: true lumen; right: blind pouch). c A greenish-yellow coagulum covered the diseased area after topical ABS application. d At last followup, 10 weeks later, complete healing of ulcerated areas is seen, while rare telangiectases and mucosal friability persisted. 


\begin{tabular}{|c|c|c|c|c|c|c|}
\hline $\begin{array}{l}\text { Patient } \\
\text { no. }\end{array}$ & $\begin{array}{l}\text { Patient age, years; } \\
\text { sex }\end{array}$ & $\begin{array}{l}\mathrm{Hb} \\
\text { (Hct) }\end{array}$ & $\begin{array}{l}\text { Details of } \\
\text { bleeding lesion }\end{array}$ & $\begin{array}{l}\text { ABS dose per session } \\
\text { (no. of sessions) }\end{array}$ & Early response* & Final result ${ }^{\dagger}$ \\
\hline 1 & $65 ; M$ & $\begin{array}{l}7.9 \mathrm{~g} / \mathrm{dL} \\
(24.2 \%)\end{array}$ & $\begin{array}{l}\text { T: grade } 3 \\
\mathrm{CM} \text { : grade } 1 \\
\mathrm{U} \text { : none } \\
\mathrm{S}, \mathrm{N} \text { : none }\end{array}$ & $20 \mathrm{~mL}(5)$ & $\begin{array}{l}\text { Bleeding stopped } \\
\text { for } 3 \text { days }\end{array}$ & No change \\
\hline 2 & $61 ; M$ & $\begin{array}{l}12.1 \mathrm{~g} / \mathrm{dL} \\
(35 \%)\end{array}$ & $\begin{array}{l}\text { T: grade } 3 \\
\text { CM: grade } 2 \\
\text { U: none } \\
\text { S, N: none }\end{array}$ & $20-30 \mathrm{~mL}(5)$ & $\begin{array}{l}\text { Bleeding stopped } \\
\text { for } 2-8 \text { days }\end{array}$ & No change \\
\hline 3 & $60 ; M$ & $\begin{array}{l}11.0 \mathrm{~g} / \mathrm{dL} \\
(33.2 \%)\end{array}$ & $\begin{array}{l}\text { T: grade } 3 \\
\text { CM: grade } 2 \\
\text { U: none } \\
\text { S, N: none }\end{array}$ & $30 \mathrm{~mL}$ (7) & $\begin{array}{l}\text { Bleeding lessened } \\
\text { for } 3 \text { days }\end{array}$ & No change \\
\hline 4 & $68 ; M$ & $\begin{array}{l}11.8 \mathrm{~g} / \mathrm{dL} \\
(34 \%)\end{array}$ & $\begin{array}{l}\text { T: grade } 3 \\
\text { CM: grade } 1 \\
\mathrm{U} \text { : none } \\
\mathrm{S}, \mathrm{N} \text { : none }\end{array}$ & $20 \mathrm{~mL}(5)$ & $\begin{array}{l}\text { Bleeding stopped } \\
\text { for } 1-3 \text { days }\end{array}$ & No change \\
\hline 5 & $56 ; F$ & $\begin{array}{l}10.1 \mathrm{~g} / \mathrm{dL} \\
(32 \%)\end{array}$ & $\begin{array}{l}\text { T: grade } 1 \\
\text { CM: grade } 2 \\
\text { U: grade } \\
\text { S, N: none }\end{array}$ & $20 \mathrm{~mL}(7)$ & $\begin{array}{l}\text { Bleeding stopped } \\
\text { for } 3-5 \text { days }\end{array}$ & $\begin{array}{l}\text { No change } \\
\text { (except healed ulcers) }\end{array}$ \\
\hline 6 & $71 ; M$ & $\begin{array}{l}12.2 \mathrm{~g} / \mathrm{dL} \\
(36 \%)\end{array}$ & $\begin{array}{l}\text { T: grade } 3 \\
\text { CM: grade } 2 \\
\text { U: grade } 3 \\
\text { S, N: none }\end{array}$ & $20 \mathrm{~mL}(5)$ & $\begin{array}{l}\text { Bleeding stopped } \\
\text { for } 1-2 \text { days }\end{array}$ & $\begin{array}{l}\text { No change } \\
\text { (except healed ulcer) }\end{array}$ \\
\hline 7 & $70 ; F$ & $\begin{array}{l}8.0 \mathrm{~g} / \mathrm{dL} \\
(24.3 \%)\end{array}$ & $\begin{array}{l}\text { T: grade } 3 \\
\text { CM: grade } 2 \\
\text { U: grade } 3 \\
\text { S, N: none }\end{array}$ & $20 \mathrm{~mL}(5)$ & $\begin{array}{l}\text { Bleeding stopped } \\
\text { for } 1-3 \text { days }\end{array}$ & $\begin{array}{l}\text { No change } \\
\text { (except healed ulcer) }\end{array}$ \\
\hline 8 & $61 ; M$ & $\begin{array}{l}11.6 \mathrm{~g} / \mathrm{dL} \\
(35.3 \%)\end{array}$ & $\begin{array}{l}\text { T: grade } 2 \\
\mathrm{CM}: \text { grade } 1 \\
\mathrm{U}: \text { grade } 3 \\
\mathrm{~S}, \mathrm{~N} \text { : none }\end{array}$ & $20 \mathrm{~mL}(5)$ & $\begin{array}{l}\text { Bleeding stopped } \\
\text { for } 3 \text { days }\end{array}$ & $\begin{array}{l}\text { No change } \\
\text { (except healed ulcer) }\end{array}$ \\
\hline
\end{tabular}

T, telangiectasia; CM, congested mucosa; $\mathrm{U}$, ulcer; S, stricture; N, necrosis (Wachter classification [3]).

* Immediate hemostasis lasting for given number of days after every session.

$\uparrow$ Final appearance of the lesion compared to initial.

\section{References}

1 Ozaslan E, Purnak T, Haznedaroglu IC. Ankaferd Blood Stopper in GI bleeding: alternative for everything? Gastrointest Endosc; in press: DOI: $10.1016 /$ j.gie.2010.05.010

2 Ozaslan E, Purnak T, Yildiz A et al. The effect of Ankaferd Blood Stopper on severe radiation colitis. Endoscopy 2009; 41 (Suppl 2): E321 - E322

3 Wachter S, Gerstner N, Goldner G et al. Endoscopic scoring of late rectal mucosal damage after conformal radiotherapy for prostatic carcinoma. Radiother Oncol 2000; 54: 11 19

4 Postgate A, Saunders B, Tjandra J et al. Argon plasma coagulation in chronic radiation proctitis. Endoscopy 2007; 39: 361 - 365

\section{Bibliography}

DOI $10.1055 / \mathrm{s}-0030-1255773$

Endoscopy 2010; 42: E271 -E272

(c) Georg Thieme Verlag KG Stuttgart · New York . ISSN 0013-726X

\section{Corresponding author}

\section{E. Ozaslan, MD}

Numune Education and Research Hospital,

Department of Gastroenterology

Cukurambar Mah. 40. Cad. 5/13

Cankaya

Ankara

Turkey

Fax: +90-312-3125026

er72@hotmail.com 